\title{
Research on Control Structure of Controllable Network System
}

\author{
Zengguang Wang \\ Department of Information Engineering \\ Mechanical Engineering College \\ Shijiazhuang, China \\ wang1223797579@163.com \\ Liyun Chen \\ Department of Information Engineering \\ Mechanical Engineering College \\ Shijiazhuang, China \\ wang1223797579@163.com
}

\begin{abstract}
The basic control structures and their structural characteristics of the controllable network system are proposed based on the introduction of controllable network. The basic control structures consist of the centralized control structure, the decentralized control structure and the hierarchical control structure. The features of control structure in the controllable network system are described. All these provide an essential basis for further research on controllable network.
\end{abstract}

Keywords-Controllable Network System; Controllable Structure; Concept; Features

\section{INTRODUCTION}

With the rapid development of the military, electronic commerce and Internet of things, the grim situation of network security proposes the urgent demand for the research of controllable network. The core idea of controllable network system is the use of automatic control theory to solve the problem of network security. Its goal is to establish a scientific and reasonable dynamic network security protection system based on the integration of existing network security technology construct efficient in order to solve network security problems [1]. One of the core issues of building the controllable network system is the network security structure, which is designed according to the characteristics of the network and the security requirement.

\section{THE CONCEPT OF CONTROLLABLE NETWORK SYSTEM}

The controllable network system is the network system which realizes the network security target through the feedback control. The controllable network system generally includes four parts: the application control part, the

\author{
$\mathrm{Yu} \mathrm{Lu}$ \\ Equipment Command and Management Department \\ Mechanical Engineering College \\ Shijiazhuang, China \\ wang1223797579@163.com \\ Wenxin Qiao \\ Equipment Command and Management Department \\ Mechanical Engineering College \\ Shijiazhuang, China \\ wang1223797579@163.com
}

controlled part, the execution unit and the feedback unit [2], and the control structure model is shown in Fig. 1.

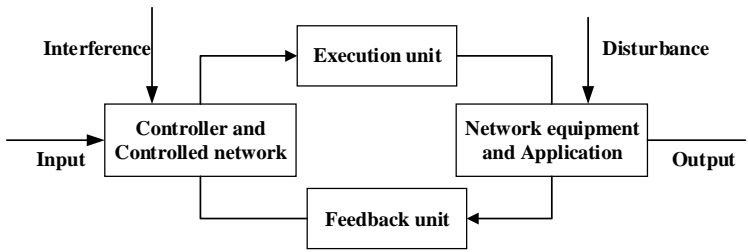

Figure 1. Control structure model of controllable network system

The network equipment and application belong to the controlled parts, including the various information systems running in the equipment and network.

The controller and controllable network belong to the implement of control section, which includes a variety of network security control personnel in the use of personnel and the network application system, the control sub network contains a variety of network control system and network management center.

The execution unit includes a variety of control and control channels. The controllable network system can change its movement and enter some kind of state under the influence of controlling effect. The significant of the controllable system is that he motion of the system reach or approach the state which is selected in the state space of all possible states of controlled system in a certain. Control channel is a combination of control structure, control mode and transmission medium of control information. On the control channel, the control information is transmitted from the control part to the controlled part and belongs to the forward channel.

The feedback unit includes the feedback effect and the feedback channel. Under the influence of the feedback, the controllable network system can monitor the status of the system. The significant of feedback is that the state of 
controlled system is monitored, analyzed, reported, in order that the control system can make timely response, the existence of feedback effects is a typical feature of cybernetics system [3]. The feedback channel is composed of various information gathering and analyzing equipment, information feedback and decision-making system. On the feedback channel, the feedback information is transmitted from the controlled part to the control part and belongs to the reverse channel.

The controllable network feedback consists of the minor loop feedback control and loop feedback control, the minor loop feedback control is in the equipment or component level to achieve the control, such as network terminal security agent, router routing control components. is characterized by itself can form the safety control loop; loop feedback control is system level implementation of the control, such as firewall, intrusion detection, authentication system and network security control center control loop, is characterized by in network security control center and the coordination and control, the system individually or jointly formed a safety control loop.

\section{THE CENTRALIZED STRUCTURE FOR CONTROLLABLE NETWORK SYSTEM}

When the network size is not too large, the network security control center and control object from the scene is near, the centralized controllable structure can be used in the occasion of high efficiency and reliability, such as the unit of general local area network (LAN) or military command and control center [4]; controllable network system of centralized control structure is shown in Fig. 2.

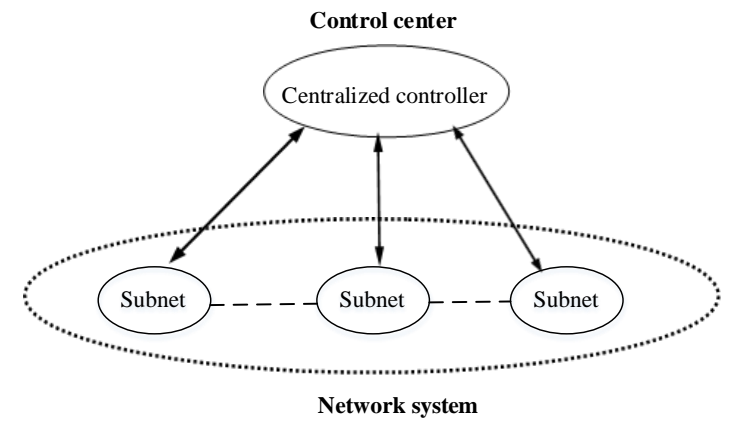

Figure 2. Centralized structure of the controllable network system

The features of centralized control structures are as follows:

With a star topology: the centralized network controller of network security control center makes a centralized control in each sub-network system of controllable network system, develop a unified control strategy, sends out the control command. Information about the running status of the subsystems in the controllable network system is centralized to the network security control center, and the unified information processing and centralized observation are centralized.

With centralized information channel structure: the global state of the centralized controller is controllable and observable in the structure. In the centralized controller and the controlled sub network, the longitudinal information flow is carried on, the upstream state observation information flow, and the control instruction information flow is issued.

The concentration of power and functions: network security control center can makes a unified, centralized observation and control to the global running state of controllable network system, the control effectiveness of controlled network system is high. In order to realize centralized control system of the controllable network, the safety control computer system is usually installed in the network security control center. The information channel of the information channel is transmitted and controlled by the controllable network and can be easily realized.

Fault concentration and risk concentration: if the security control center centralized controller is faulty, the controllable network system will be paralyzed. It will lead to lower structure and reliability of the system operation, we must consider the safety control question of core system backup. When the scale of the controllable network system is large, it is difficult to adopt centralized control structure.

In the centralized control structure, the control and observation information channels between the centralized controller and the network terminal or the sub network are formed by the star shaped topology, as shown in Fig. 3.

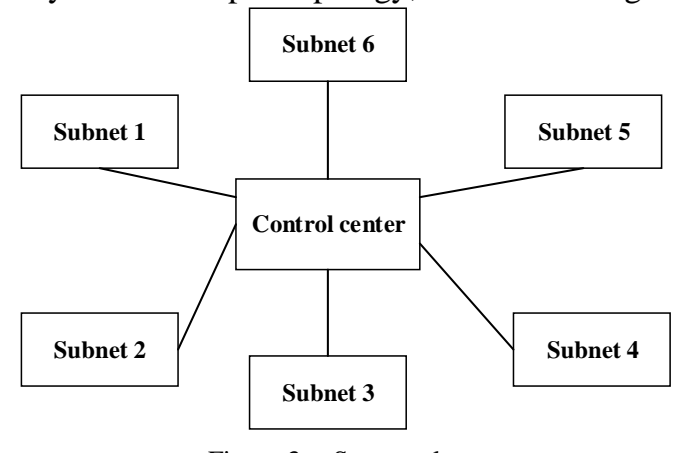

Figure 3. Star topology

\section{THE DECENTRALIZED STRUCTURE FOR CONTROLLABLE NETWORK SYSTEM}

When the system of the controllable network scale is too big, It is difficult to centralized control. The decentralized control structure can be used in the occasion that the coordination of system requirements is not high, or communicate with each other more convenient, such as the Internet system. The decentralized control structure of controllable network system of decentralized control structure is shown in Fig. 4. 


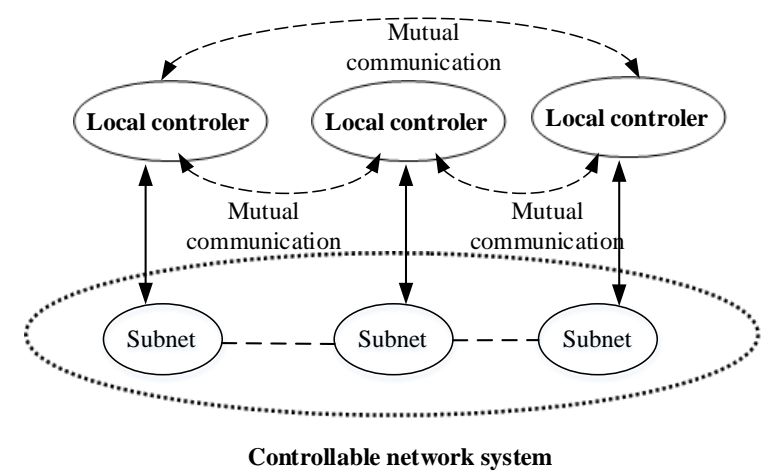

Figure 4. Decentralized structure of the controllable network system

The characteristics of decentralized control structures are as follows [5] [6]:

Insider control and the synergies between the interoffice: The centralized control structure can be used when separating the local controller control sub network; because there is no centralized global controller, for the coordination between the local controllers of sub network, each dispersed among local controller needs mutual communication and mutual coordination to complete the task of overall control.

The logic structure was determined to control structure: From the perspective of the physical topology, decentralized local controller can be connected to each other, but the control structure of the cooperative control is determined by the logical topology.

With relatively decentralized information channel structure: The control and observation of the sub network are carried out independently by a number of local controllers; each local controller can only control and observe the corresponding local sub network, send out the local control instruction and receive the local observation information; the global state of the local decentralized controller is uncontrollable and unobservable in the structure.

Decentralized fault, risk diversification: because of the structure of information channel, the global failure of the controllable network system can be caused even when a certain controller fails, so the controllable network system has high reliability. The task of each local controller is relatively simple and easy to implement and can be installed nearby, facilitating the observation and control signal transmission. The local control and observation information transmission equipment is simple to obtain timely observation information and the formulation of control strategy, sends out the control command. It is highly effective and good flexibility to the corresponding sub network control.

The uncontrollable and unobservable of global structure: local controller to control network system of global state is uncontrollable and unobservable because of not centralized information channel structure. The mutual relationship between the sub networks, the state observation and the state control are the interaction. It is thus necessary to coordinate between multiple local decentralized controllers that rely on mutual communication coordination, communication time delay and disturbance. It is difficult to comprehensive, timely coordination, effectiveness coordination. So the control for networked systems with global control is lower.

When the controllable network system adopts the distributed control structure, since there is no global coordination controller, it can only rely on the mutual communication between the system to realize controllable network system of cooperative control, the scattered controller between mutual communication can take the following methods.

\section{A . Master-Slave Type Coordination}

A sub network is selected as the main sub network, while the other sub networks are subordinate sub networks. By the dominant sub network of the subordinate sub network working state of observation and evaluation, a collaborative signal and of the subordinate sub network synergetic control and the control characteristics and the hierarchical control system is the same, the dominant sub network is in hierarchical control system coordination device. Under the guidance of the leading sub network, the total and the total tasks of the network system are realized. The structure of its communication is shown in Fig. 5 (Fig. 1 of the sub network is the sub network, the sub network 2, 3, 4 are subordinate sub network).

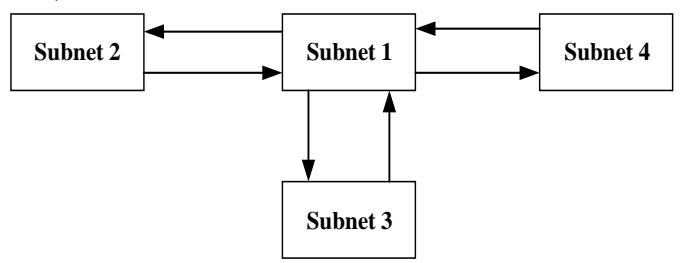

Figure 5. Master-slave type cooperative structure

The master-slave type cooperative network topology is the star topology structure, which can choose one node or the subnet as the dominant, the other is subordinate.

\section{B. The Cyclic Type Coordination}

The structure of the decentralized control structure is shown in Fig. 6. The status of each sub network is equal in the cyclic type cooperative network control scheme. But in a sequence, in serial mode, the cyclic type is coordinated in turn. The loop can be clockwise and it can also be an anticlockwise circulation, in the collaborative network control loop and the adjacent sub networks. It is both collaborative and coordinated, and its control characteristics are similar to the token ring network.

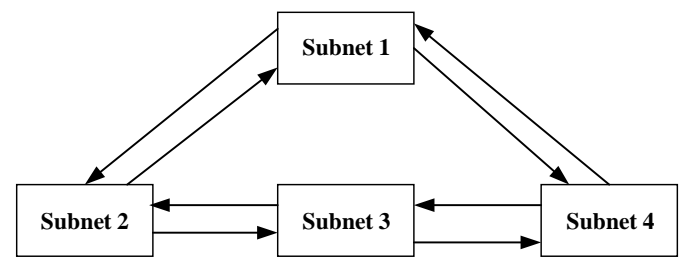

Figure 6. Cyclic type cooperative structure

The network topology is cooperative cyclic ring topology network topology, no master-slave points. 


\section{General Formula of Coordination}

The so-called "general collaborative" refers to the collaborative structure diagram of control network is fully connected graph, can fully communicate with each other. The structure of the general collaborative structure is shown in Fig. 7.

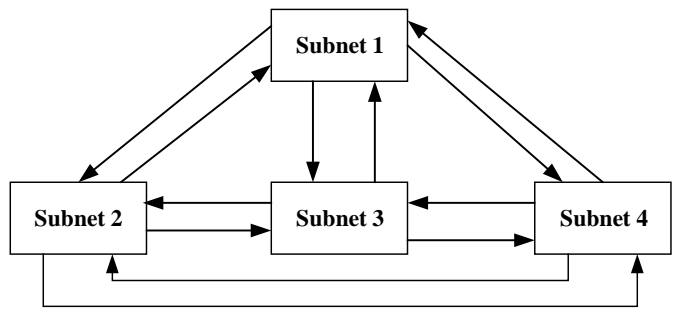

Figure 7. General collaborative structure

There is two-way collaborative structure between any two sub networks and the two-way collaboration can be carried in all pass collaborative decentralized control scheme. Therefore, any sub network is both the collaborators and the collaborators. The sub-network in the process of sub-goals and sub-task is achieved in each sub-network of mutual collaboration, mutual cooperation and mutual restraint through the whole formula collaboration. At the same time, the overall objective of the network system and general task of network system are achieved. The network topology is the general collaborative bus topology.

\section{Grouping Type Coordination}

According to the strong and weak of the coupling between the subsystems and the difficulty of communication and the difference of the cooperative demand, the sub network is divided into some groups. The cooperative scheme of the cooperative scheme, such as master-slave type, cyclic type and so on, can be used in the coordination of the cooperative scheme and the cooperative scheme [7]. The network topology of packet type is the hybrid topology of network topology, as shown in Fig. 8.

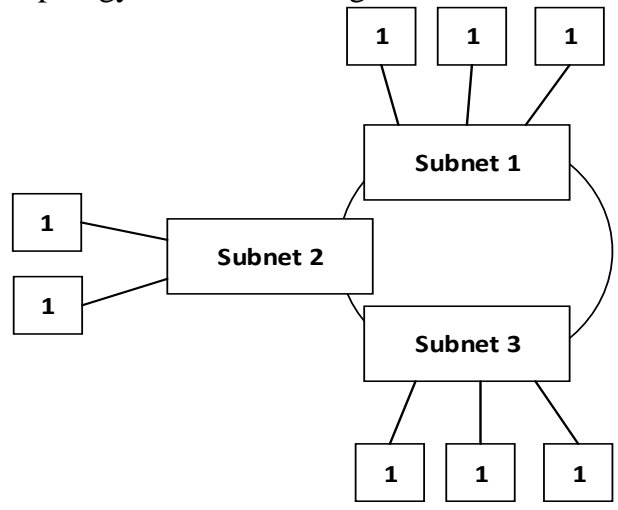

Figure 8. Hybrid topology

The above four kinds of cooperative schemes are typical cooperative control schemes for decentralized network systems. In the practical network system, they can be combined with each other flexibly. For example, for the size of the huge decentralized network system, the direct use of the whole formula of synergistic programs may be difficult to achieve, at this time, available grouping cooperative scheme can be used. Each packet of cooperative network control, according to the specific conditions, can be flexible application of master-slave coordination, circulation collaborative or full general cooperative scheme; Masterslave coordination, circulation collaborative and general cooperative scheme can be selected in group synergy.

\section{HIERARCHICAL STRUCTURE FOR CONTROLLABLE NETWORK SYSTEM}

The hierarchical control structure of the controllable network system is shown in Fig. 9. Taking the two level hierarchical control systems as an example, the subordinate parts of the controllable network system are composed of $\mathrm{N}$ local control sub network. The supervisor is the cooperative network. Collaborative network control task is to properly handle the relationship among sub networks, in each sub network of local optimization based, through collaborative, network system optimization [8].

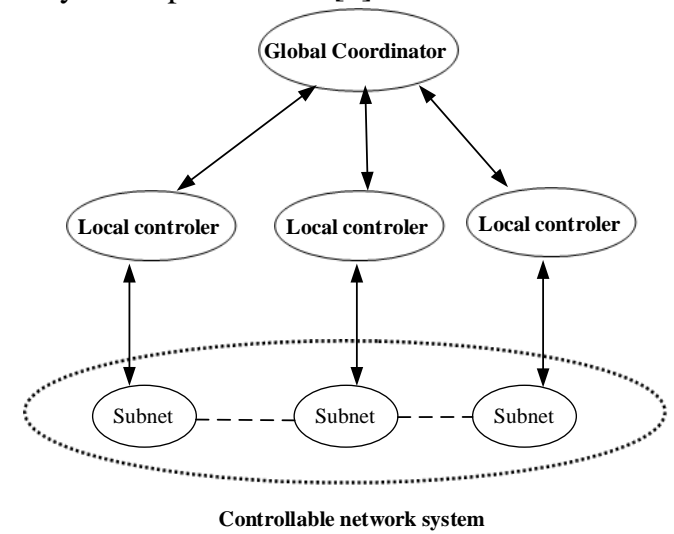

Figure 9. Hierarchical structure for controllable network system

Hierarchical network system of cooperative control is divided into two steps: one is decomposed. The problem of local optimal control for each sub network is decomposed some simple sub network and the local optimal control problem is solved by the appropriate processing of the complex network system. The other is coordination: Through Model Co target collaboration, on the basis of the local optimal network, it is easy to achieve the global optimum of network system.

The structure of hierarchical control structure is as follows[9]:

The hierarchical control structure has the hierarchical information channel structure, the information channels between the global coordinator and the local controller, the sub network are formed, and the tree topology is formed. The global coordinator can control and observe the global state of the network system indirectly by local controllers.

The hierarchical control structure is adopted for hierarchical control structure. Among them, the local decentralized controllers, respectively of corresponding sub network of local control and observation; global coordinator 
through in every local controller coordinated control and coordinated observations and indirectly on the network system of global and centralized control and global observation, so as to realize the "concentration to dispersion" combination of network system hierarchical control and dispersion concentration "combination of controllable network system based on hierarchical observation.

For the hierarchical control structure for the "global Coordinator - local controller - sub network", the hierarchical transfer of longitudinal information flow, among them, between the "global coordinator, local controller, the transfer is global coordinator of the coordinated control and coordinated observations of information; between the local controller, sub network, the transfer is between the local coordinator and local sub network control and observation information.

The hierarchical control structure of centralized control and decentralized control combination, both dispersed, direct and timely local control, and centralized, indirect and global coordination control, both centralized control and decentralized control. Therefore, local control of global coordination of network system and the sub network high effectiveness; local controller failure affects only the corresponding local sub network, equivalent to decentralized control of reliability. Global coordinator failure will lead to global coordination failure, but the local controllers can still continue to run, hierarchical control will degenerate into a decentralized control, network system not as completely paralyzed, so that the operation of the high reliability.

The hierarchical control system has the quasi centralized information structure, which is controllable and observable in structure. Each local controller and the corresponding sub object nearest to install, easy to local control and observation signal transmission; coordinator only coordinated control without direct global control of network system, task coordination is relatively simplified, less coordinated control and coordinated observations of the amount of information, but also easy to transmission and processing.

Therefore, the hierarchical control can make up for the disadvantages of centralized control and decentralized and has advantages of both. The hierarchical control structure has been widely used and it is the generally applicable control structure in various fields.

\section{THE FEATURES OF CONTROL STRUCTURE IN CONTROLLABLE NETWORK SYSTEM}

\section{A. Similarity}

The controllable network system consists of many small networks associated with each other. The controllable network system can be decomposed into several small networks by the organization and the physical. Therefore, the study of the similarity of network is conducive to the analysis of the network system. For example, in the stability analysis of the network, the network is stable, whether the composition of the network system is stable; if there is a small and unstable network, the stability of the network can be formed. The requirements to the interrelated aspects of stability can be putted forward by similarity analysis of network stability in order to seek stable conditions to ensure network systems and ways to improve network stability. Similarly, the observability and controllability of the similar network are also analyzed.

\section{B. Dynamics}

The controllable network is in dynamic flux, its structure and parameters are constantly changing. The dynamic performance is mainly reflected in the following two aspects: the dynamic of the host access and the the dynamics of the network behavior. The host access can happen at anytime and anywhere because of the structure of the network system becoming more and more complex. There are many uncertainties in network system consisting of the subjective aspects and the objective aspects ${ }^{[10]}$.

\section{Compound}

The result of the structure analysis shows that the basic control structure consist of the centralized control structure, the decentralized control structure and the hierarchical control structure in the network system, although tha actual network structure is very complex. The hierarchical control structure is commonly used control structure and a combination of the centralized control structure and the decentralized control structure. The actual structure of the various network system can be regarded as a controllable conbination, modifications and extensions of the three basic structure.

\section{CONCLUSION}

The design of network security control is the core of controllable network system. The network security control structure of network security control center has great influence on controllable network system security, reliability, controllability and effectiveness. The network security control structure is selected according to the situation of the network size, as well as on the requirements of network performance and the network safety.

\section{REFERENCES}

[1] $\mathrm{Lu} \mathrm{Yu,} \mathrm{Wang} \mathrm{Yu,Wu} \mathrm{Zhongwang.} \mathrm{Security} \mathrm{Control} \mathrm{for} \mathrm{Info-Net[M]}$ Beijing: National Defense Industry Press ,2011.

[2] He Ning, Lu Yu, Wang Lei. Application of Network Control Theory in Network Attack and Defense [J]. Wuhan Univ.(Nat.Sci.Ed),2006, 52(5):639-643.

[3] Chi Ming. Analysis and Designs for Feedback Control System under Network Circumstance[D].Guangzhou : South China University of Teachnology, 2013.

[4] Lu Fei, Cheng Peixing, Yin Qiyu. A Service Composition Method of Command and Control System with Distributed Data Flow[J]. Command Control \& Simulation 2012, 34 (4) :94-97.

[5] YU Tian.Reserchching on Dechentralized Control Computer Interlocking System Network Communication[D].Chengdu: Southwest Jiaotong University,2010.

[6] WANG Rong. Analysis for Distributed Control of Dynamical Networks[D].Zhuzhou:Hunan University of Technology,2013.

[7] $\mathrm{Lu} \mathrm{Yu}$. Introduction to Network Control Theory[M].National Defence Industy Press,2005.

[8] Xu Jia, Feng Guodeng, Su Purui. Research on Network-Warning Model Based on Dynamic Peer-to-Peer Overlay Hierarchy[J]Journal of Computer Research and Development,2010.47(9):1574-1586. 
[9] [9] Song Yun-Zhong and Tang Yi-Fa. Hierarchical-control-based output synchronization of coexisting attractor networks $[\mathrm{J}]$. Chin.Phys.B, 2010,19(2):020506.
[10] [10] DAI Bin, WANG Hang-yuan, XU Guan, YANG Jun. Opportunities and Threats Coexist in SND Security[J]. Application Research of Computers,2014,31(8):2254-2262. 